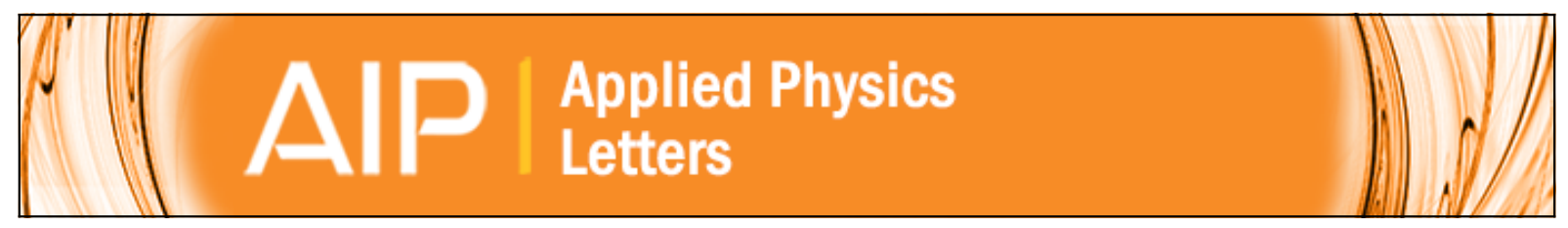

\title{
Substituent position-induced color tunability in polymer light-emitting diodes
}

Jin Young Kim, Sung Heum Park, Kwanghee Lee, In Suk Yum, and Sung Ho Jin

Citation: Applied Physics Letters 81, 1732 (2002); doi: 10.1063/1.1502911

View online: http://dx.doi.org/10.1063/1.1502911

View Table of Contents: http://scitation.aip.org/content/aip/journal/apl/81/9?ver=pdfcov

Published by the AIP Publishing

\section{Articles you may be interested in}

Color tuning and efficiency enhancement through interfacial energy transfer and thickness control in lightemitting diodes

J. Appl. Phys. 96, 2272 (2004); 10.1063/1.1765857

Improving the performance of polymer light-emitting diodes using polymer solid solutions

Appl. Phys. Lett. 79, 578 (2001); 10.1063/1.1389324

Efficient light-emitting diodes based on a binaphthalene-containing polymer

Appl. Phys. Lett. 75, 3745 (1999); 10.1063/1.125442

Naphthalimide side-chain polymers for organic light-emitting diodes: Band-offset engineering and role of polymer thickness

J. Appl. Phys. 83, 2343 (1998); 10.1063/1.366977

Novel main-chain poly-carbazoles as hole and electron transport materials in polymer light-emitting diodes Appl. Phys. Lett. 71, 1921 (1997); 10.1063/1.119981

A|P|Applied Physics

AlP | Letters

is pleased to announce Reuben Collins as its new Editor-in-Chief

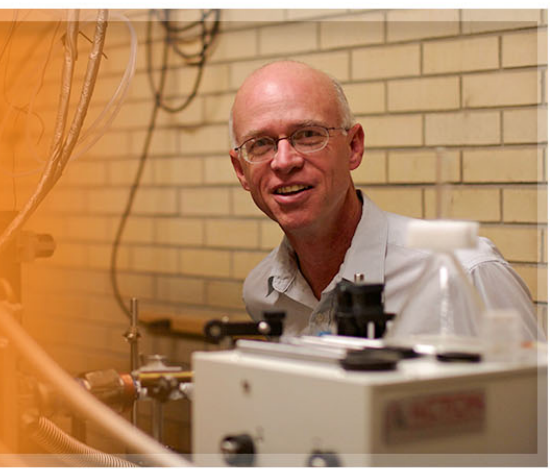




\title{
Substituent position-induced color tunability in polymer light-emitting diodes
}

\author{
Jin Young Kim, Sung Heum Park, and Kwanghee Lee ${ }^{\text {a) }}$ \\ Department of Physics, Pusan National University, Pusan 609-735, Korea \\ In Suk Yum and Sung Ho Jin \\ Department of Chemistry, Pusan National University, Pusan 609-735, Korea
}

(Received 11 February 2002; accepted for publication 1 July 2002)

\begin{abstract}
We report substituent position-induced color tunability in polymer light-emitting diodes fabricated with poly[2-\{2- or 3- or 4-[(3,7-dimethyloctyl)oxy] phenyoxy\}-1,4-phenylenevinylene] (DMOPPPV). When the position of the substituent in DMOP-PPV moves to the ortho, meta, and para sites, the corresponding photo- and electroluminescence spectra shift their peaks to a longer wavelength of about 540,560 , and $585 \mathrm{~nm}$, respectively. We ascribe this to the different degree of steric effect in the backbone for each substituent position. As the substituent position is closer to the main chain, the planarity of the backbone is less conserved, thereby reducing the effective conjugated length of the main chain and broadening the $\pi-\pi^{*}$ energy gap. (C) 2002 American Institute of Physics.
\end{abstract}

[DOI: $10.1063 / 1.1502911]$

Polymer light-emitting diodes (LEDs) continue to be of interest because of the potential application in flat-panel displays. ${ }^{1}$ The main advantage of using soluble semiconducting polymers as active emitting materials is a color tunability over a wide spectral range together with easy processing and mechanical flexibility. Since the first polymer LED based on poly(p-phenylenevinylene) (PPV) in $1990,{ }^{2}$ a variety of materials have been developed as luminescent polymers to span the entire visible spectrum. ${ }^{3}$ Conjugated polymers usually achieve color tuning by altering the chemical structure of the main chain itself, ${ }^{4}$ or by introducing various substituents into the backbone. ${ }^{5}$ In such a case, a polymer with a different chemical structure (that is, with a different emission color) is expected to possess a different chemical processibility. This might make the fabrication process complicated in the future application of these systems to full color displays, and also acts as a major drawback in fabricating blend systems for white light sources using those polymers.

Another alternative approach to color tuning in polymer systems is to adjust the effective conjugation length, for example, by controlling the chain length ${ }^{6}$ or degree of regioregularity ${ }^{7}$ in polymer systems. Recently it was also pointed out that control of the film morphology induces a change in the band gap of polymers. ${ }^{8}$ In fact, it is quite well known that the degree of effective conjugation length determines the magnitude of the $\pi-\pi^{*}$ energy gap in conjugated polymer systems. ${ }^{9}$ In this study we present substituent position-induced color tunability in PPV derivatives, poly[2$\{2-$ or 3- or 4-[(3,7-dimethyloctyl)oxy]phenyoxy $\}-1,4-$ phenylenevinylene] (DMOP-PPV). By simply controlling the position of the substituent, 3,7-dimethyloctyloxy group, in the ortho (o-), meta (m-), and para (p-) sites of the phenyl side ring in DMOP-PPV as shown in Fig. 1, we expect a different degree of steric hindrance along the $\pi$-conjugated

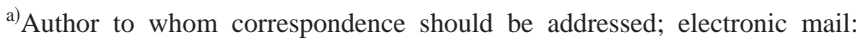
kwhlee@pusan.ac.kr
}

polymer backbone. This induces a change in the ring torsion angle toward coplanarization along the backbone, resulting in a different degree of the effective conjugation length for each substituent site.

Three kinds of DMOP-PPV samples each with different substituent position (o-, m-, and p-DMOP-PPV) were synthesized by the Gilch polymerization method ${ }^{10}$ as described in detail elsewhere. ${ }^{11}$ Each material was dissolved in chlorobenzene $(\mathrm{CB})$ with a concentration of $0.5 \mathrm{wt} \%$, and spin coated on a precleaned fused silica with a thickness of around $80 \mathrm{~nm}$. Absorption spectra were recorded by a Varian 5E UV-VIS-NIR spectrophotometer, while the Oriel InstaSpec IV CCD detection system in combination with a typical integrating sphere technique was used for the photoluminescence (PL) and electroluminescence (EL) spectra, and also for the external quantum efficiency measurements. ${ }^{12}$

Utilizing those three DMOP-PPV materials as emitting layers, we fabricated typical polymer LEDs with a structure of ITO/PEDOT:PSS/DMOP-PPV/Al. The glass substrates coated with indium tin oxide (ITO; $<20 \Omega / \square$ ) were thoroughly cleaned by a wet cleaning process in an ultrasonic bath and heated in a vacuum dry oven. Commercially available poly(ethylene-dioxy)thiophene mixed with poly(stylene-sulfonic acid), PEDOT:PSS, was used as a buffer layer $(\sim 40 \mathrm{~nm})$, and spin coated onto the precleaned ITO substrate with curing at $100^{\circ} \mathrm{C}$ in the vacuum dry oven for an hour. The emitting polymer layer was spin coated on top of the PEDOT:PSS layer with a solution of $0.5 \mathrm{wt} \%$ in CB. Then, a $100 \mathrm{~nm}$ thick aluminum layer as the cathode

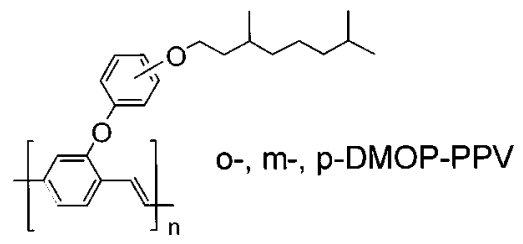

FIG. 1. Chemical structure of o-, m-, and p-DMOP-PPV. 

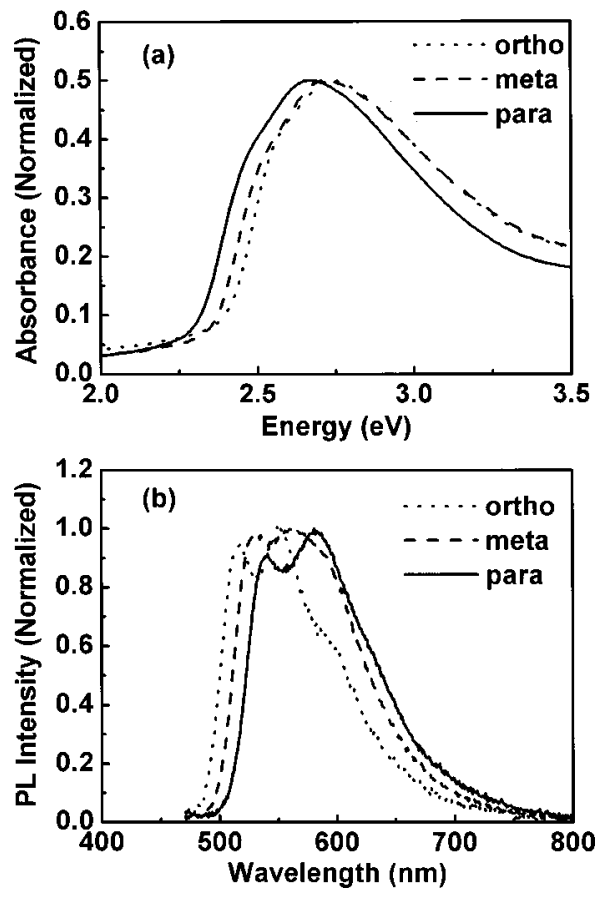

FIG. 2. Absorption (a) and PL spectra (b) of o-, m-, and p-DMOP-PPV.

was deposited by thermal evaporation in a vacuum of about $2 \times 10^{-5}$ Torr. Current density-voltage $(J-V)$ and luminance-voltage $(L-V)$ characteristics of all the devices were measured using a Keithley 236 Source Measure Unit equipped with a calibrated photomultiplier tube.

Figure 2 shows the absorption and PL spectra of the DMOP-PPV samples. When the substituent position changes from ortho- to meta- and para-site, the absorption edge moves to a longer wavelength corresponding to $2.41,2.37$, and $2.31 \mathrm{eV}$, respectively. This observation is also consistent with the PL spectra. The emission peak of p-DMOP-PPV is observed at a longer wavelength of $585 \mathrm{~nm}$ with pronounced vibronic features, as compared with those of o-DMOP-PPV $(549 \mathrm{~nm})$ and m-DMOP-PPV $(560 \mathrm{~nm})$.

Figure 3 shows the electroluminescence (EL) spectra of $\mathrm{o}-, \mathrm{m}-$, and $\mathrm{p}-\mathrm{DMOP}-\mathrm{PPV}$, also shifting their main peaks to 541, 560, and $586 \mathrm{~nm}$, consistent with the absorption and PL spectra measurements. This wavelength range corresponds to the color from green to orange-red. When these EL spectra are converted into chromaticity coordinates on a CIE (Commission Internationale de l'Eclairage) 1931 diagram, one finds $\mathrm{x}=0.33$ and $\mathrm{y}=0.57$ for o-DMOP-PPV, $\mathrm{x}=0.38$ and

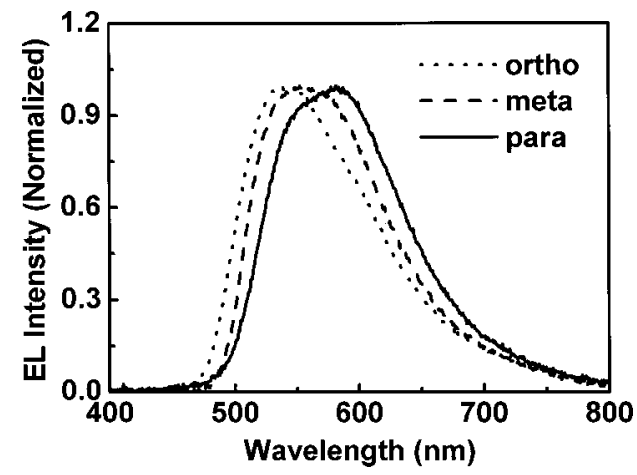

FIG. 3. Normalized EL spectra of the devices using 0 -, m-, and p-DMOPPPV with a configuration of ITO/PEDOT:PSS/DMOP-PPV/Al.

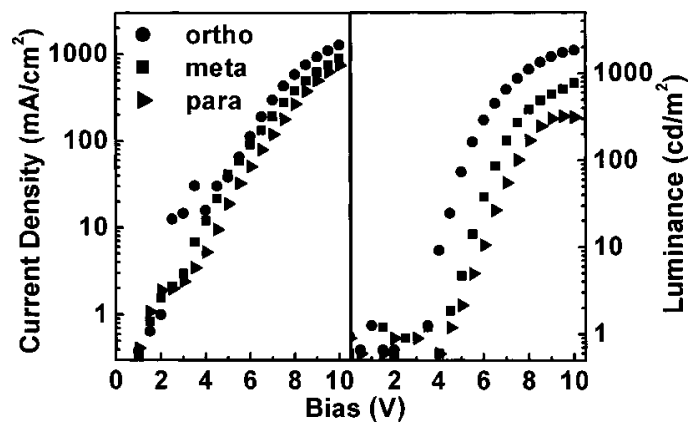

FIG. 4. Current density-voltage and luminance-voltage characteristics of the typical ITO/PEDOT:PSS/DMOP-PPV/Al devices.

$\mathrm{y}=0.58$ for $\mathrm{m}$-DMOP-PPV, and $\mathrm{x}=0.41$ and $\mathrm{y}=0.54$ for p-DMOP-PPV, respectively.

Considering that such color tuning is induced only by the position of the substituent without any chemical change in the polymer systems, we attribute this to the deviation from coplanarity of the conjugated backbone for each substituent position. As the position of the 3,7-dimethyloctyloxy group moves from the para- to the ortho-site in the phenoxy side group (see Fig. 1), steric hindrance between the 3,7dimethyloctyloxyphenoxy side group and vinylic protons in the main chain gives rise to a distortion in the coplanarity of the polymer backbones. We believe that this reduces the effective conjugated length of the main chain and broadens the corresponding $\pi$ - $\pi^{*}$ energy gap.

Another noticeable aspect is the high value of the external PL quantum efficiency for these systems. According to our measurements employing a typical integrating sphere method, ${ }^{12}$ the values of o-, m-, and p-DMOP-PPV are as high as $43 \% \pm 1 \%, 54 \% \pm 1 \%$, and $40 \% \pm 2 \%$, respectively. These values are almost four or five times higher than that of MEH-PPV ( $\sim 10 \%$ in our measurement). We interpret that this might be associated with the bulky nature of the side group of DMOP-PPV, inhibiting intermolecular interaction of the polymer backbone and thereby increasing the PL efficiency as consistent with previous work. ${ }^{13}$ Considering that our structural analysis confirms the longest intermolecular distance for m-DMOP-PPV and the shortest for p-DMOP-PPV ${ }^{11}$ this interpretation is also consistent with the observation that the m-DMOP-PPV exhibits the highest PL efficiency among the three samples. In such a case, the reduced packing of the polymer chains in the m-DMOP-PPV system is responsible for the enhancement of PL efficiency as compared with the other two polymers. ${ }^{14}$

Figure 4 shows the $J-V$ and $L-V$ characteristics of the ITO/PEDOT:PSS/DMOP-PPV/Al devices. The turn-on voltage for the current injection is about $4 \mathrm{~V}$ for all devices, while that of light emission varies from 4 to $6 \mathrm{~V}$ for o-, $\mathrm{m}$ and p-DMOP-PPV. Although the effective conjugation length of o-DMOP-PPV is shorter than that of the other two polymers, the devices using o-DMOP-PPV show a better performance than those using $\mathrm{m}$ - and p-DMOP-PPV. The brightness for o-DMOP-PPV is higher with $1840 \mathrm{~cd} / \mathrm{m}^{2}$, as compared with $770 \mathrm{~cd} / \mathrm{m}^{2}$ for m-DMOP-PPV and $316 \mathrm{~cd} / \mathrm{m}^{2}$ for p-DMOP-PPV at $10 \mathrm{~V}$. Also the $J-V$ curves imply the lowest value of bulk resistance for o-DMOP-PPV (the highest value for p-DMOP-PPV), which is in contrast to the effective conjugation length consideration. Although the rea- 
son is not clear at this stage, we assume that the substituent in DMOP-PPV influences the transport properties of the injected charges in a way different from the effective conjugation length.

In summary, we have developed a conjugated polymer DMOP-PPV derivative system, in which the band gap can be tuned by changing the position of the substituent in the side group. Incorporating those materials as active layers of the polymer LEDs, we demonstrate that the emitting color can be tuned from green to orange-red. We attribute this to the different degree of steric effect in the PPV backbone for each substituent position. Furthermore, these materials show an enhanced processibility and device performance inherent in this system. Our results, therefore, demonstrate that this new class of polymers provides a strategic approach to the design and construction of promising electroluminescent materials with easy color tunability.

This work was supported by the University Research Program of the Ministry of Information and Communication in South Korea (2001-085-3).
${ }^{1}$ R. H. Friend, R. W. Gymer, A. B. Holmes, J. H. Burroughes, R. N. Marks, C. Taliani, D. D. C. Bradley, D. A. Dos Santos, J.-L. Brédas, M. Lögdlund, and W. R. Salaneck, Nature (London) 397, 121 (1999).

${ }^{2}$ J. H. Burroughes, D. D. C. Bradley, A. R. Brown, R. N. Marks, M. Mackay, R. H. Friend, P. L. Burn, and A. B. Holmes, Nature (London) 347, 539 (1990).

${ }^{3}$ A. Kraft, A. C. Grimsdale, and A. B. Holmes, Angew. Chem. Int. Ed. Engl. 37, 402 (1998).

${ }^{4}$ T. Ahn, M. S. Jang, H. K. Shim, D. H. Hwang, and T. Zyung, Macromolecules 32, 3279 (1999).

${ }^{5}$ N. C. Greenham, S. C. Moratti, D. D. C. Bradley, R. H. Friend, and A. B. Holmes, Nature (London) 365, 628 (1993).

${ }^{6}$ M. Catellani, S. Luzzati, R. Mendichi, A. G. Schieroni, and P. C. Stein, Polymer 37, 1059 (1996).

${ }^{7}$ T.-A. Chen, X. Wu, and R. D. Rieke, J. Am. Chem. Soc. 117, 233 (1995).

${ }^{8}$ Y. Shi, J. Liu, and Y. Yang, J. Appl. Phys. 87, 4254 (2000).

${ }^{9}$ K. C. Rustagi and J. Ducuing, Opt. Commun. 10, 258 (1974).

${ }^{10}$ H. G. Gilch and W. L. Wheelwright, J. Polym. Sci., Part A: Polym. Chem. 4, 1337 (1966).

${ }^{11}$ S. H. Jin, I. S. Yeom, K. Lee, J. Y. Kim, Y. S. Gal, D. K Moon, and S. P. Lee (unpublished).

${ }^{12}$ J. C. de Mello, H. F. Wittmann, and R. H. Friend, Adv. Mater. 9, 230 (1997)

${ }^{13}$ T.-Q. Nguyen, V. Doan, and B. J. Schwartz, J. Chem. Phys. 110, 4068 (1999).

${ }^{14}$ S. Son, A. Dodabalapur, A. J. Lovinger, and M. E. Galvin, Science 269, 376 (1995). 